\title{
SIMULTANEOUS EXTRACTION AND RAPID HPLC BASED QUANTIFICATION OF CROCIN AND SAFRANAL IN SAFFRON (CROCUS SATIVUS L.)
}

\author{
PRAVEJ ALAM1 ${ }^{1,}$, SHEREEN F. ELKHOLY ${ }^{1,2}$, MUNETAKA HOSOKAWA ${ }^{3}$, SABRY A. MAHFOUZ ${ }^{4}$, MAHMOUD A. \\ SHARAF-ELDIN ${ }^{1,4}$
}

1Sara Alghonaim Research Chair (SRC), Biology Department, College of Science and Humanities, Prince Sattam bin Abdul-Aziz University (PSAU), 11942 Alkharj, Kingdom of Saudi Arabia (KSA), ' 2 Plant Transformation and Biopharmaceuticals Lab, Agricultural Genetic Engineering Research Institute (AGERI), Agricultural Research Centre (ARC), Giza, Egypt, ${ }^{3}$ Graduate School of Agriculture, Kyoto University, Sakyo-ku, Kyoto, 606-8502, Japan, ${ }^{4}$ Department of Medicinal and Aromatic Plants Research, National Research Centre (NRC). 12622-Cairo, Egypt Email: alamprez@gmail.com

Received: 11 Apr 2016 Revised and Accepted: 23 Aug 2016

\section{ABSTRACT}

Objective: Saffron (Crocus sativus L.) is one of the most valuable crops with high medicinal values used in many diseases. The aim of the study was to establish the reliability and rapid HPLC method for analysis of crocin and safranal concentration present in stigmata of saffron in the market as well as field grown samples.

Methods: Field grown and market stigmata of saffron were used for extraction of crocin and safranal. The linear dynamic ranges were established after validating the robustness of critical method parameters. The Agilent1260-Infinity Quaternary LC system was used for the preparation of calibration standards and quantification of crocin and safranal in C. sativus stigmata.

Results: A good linearity was achieved in the range of 10-30 $\mu$ g for each compound with the determination coefficient $\left(\mathrm{R}^{2}\right)$. The calibration curves revealed linear regression (r. 0.997) for this rapid HPLC method and limit of quantifications (LOQs) were achieved in the range of $3.4 \mu \mathrm{g} / \mathrm{ml}$ for crocin and $10.2 \mu \mathrm{g} / \mathrm{ml}$ for safranal. The limit of detection (LODs) for all standards was $\leq 4.2 \mu \mathrm{g} / \mathrm{ml}$. The range of crocin content (10.43-16.32 mg/g) and safranal (5.19-5.21 $\mathrm{mg} / \mathrm{g})$ was estimated in saffron samples.

Conclusion: This method may serve the purpose of accurate quantification of crocin and safranal present in the stigmata of the plant in a quick time period.

Keywords: Saffron, Crocin, Safranal, Extraction, Quantification, HPLC

(c) 2016 The Authors. Published by Innovare Academic Sciences Pvt Ltd. This is an open access article under the CC BY license (http://creativecommons.org/licenses/by/4. 0/) DOI: http://dx.doi.org/10.22159/ijpps.2016v8i10.12172

\section{INTRODUCTION}

Saffron is a valuable cash crop cultivated in semiarid, arid region of the world. It is likewise cultivated in some other region like temperate and tropical climates. Saffron (Crocus sativus L.) is used as most expensive spices worldwide. The cost of the saffron is fluctuating every year due to the demand of saffron stigma. The quality of saffron depends on its aroma. It is due to the presence of the 150 volatile compounds in stigmata of the plants. These compounds are biosynthesized in the stigmata of the $C$. sativus and the aroma of saffron comes from monoterpene based compound. About $>60 \%$ essential oil is biosynthesized in the stigmata parts of the C. sativus [1]. Saffron quality depends on its major metabolites like crocin, safranal, etc., responsible for saffron's colour, taste, odour. The amount of these compounds in dried stigmata tissues is the most important indicator of quality. These compounds check the cost of saffron in the global market [2-4]. The chemical constituents of saffron were strongly dependent on drying, extraction and quantitative analysis of crocin and safranal. Safranal is converted by picrocrocin through enzymatic or thermal degradation during the drying of the stigmata [5].

In the recent past, many methods have been used like thin layer chromatography, gas chromatography, near infrared (NIR), etc., for the extraction and quantification of the safranal and crocin from the stigmata of $C$. sativus $[1,6-8]$. Some methods are non-specific and unable to adequately separate the exact quality of saffron present in the international market on the basis of metabolites. Either they are more expensive or time-consuming [9-12].

Keeping in view, we have optimized the high performance liquid chromatography (HPLC)-based method to estimate the crocin and safranal content in methanol and water extract of $C$. sativus stigmata. The objective of this study is mainly to focus on estimation of crocin and safranal level in the market as well as field grown samples influenced by either environment or regional climates.

\section{MATERIALS AND METHODS}

\section{Plant materials}

The three types of plant materials of saffron stigmata were obtained from the local market (two) and experimental farm of Sara Alghoniam Research Chair, Prince Sattam bin Abdulaziz University, Alkharj, KSA, $24^{\circ} 8^{\prime}$ 54" North, $47^{\circ} 18^{\prime} 18^{\prime \prime}$ East altitude of Saudi Arabia.

\section{Chemicals and reagents}

All the chemicals like standard crocin, safranal, methanol, and ethanol were also procured from the same company (Sigma, USA). All the solvent and water has been used in this study denoted as HPLC grade MilliQ distilled water was used after filtering through a $0.45-\mu \mathrm{m}$ filter before use through an ultra-purification water filtration system (MilliQ, USA).

\section{Extraction of crocin and safranal}

Fifteen milligrams each saffron stigmata used for extraction of these metabolites were suspended in $10 \mathrm{ml}$ of ethanol, water, methanolwater (50:50, v/v) and magnetically stirred for $24 \mathrm{hr}$ at room temperature in the dark. After extraction of crocin and safranal, samples were filtered through $0.25 \mu \mathrm{m}$ pore size filter membrane (Millipore, USA) and stored at $4{ }^{\circ} \mathrm{C}$ for HPLC analyses.

\section{HPLC equipment}

Rapid HPLC analysis was performed in a multi-solvent Agilent1260Infinity Quaternary LC system consists of quaternary pump (G131B) with high-performance auto sampler (G1367E) and thermostat coupled with Diode Array detector (DAD, noise levels of $< \pm 0.6$ $\mu \mathrm{AU} / \mathrm{cm}$ the revolutionary $6 \mathrm{~cm}$ flow cell gives up to 10 times higher sensitivity than other instruments) assembled with high accurate computer system (Dell Computer system). Agilent Open LAB 
ChemStation version C.01.05 (Agilent, USA) was used for data acquirement and processing of the chromatogram on the basis of area and retention time.

An RP-C18 column (Agilent eclipse Plus, $4.6 \mathrm{~mm}$ X $100 \mathrm{~mm}$, with a pore diameter of $95 \AA, 1.8 \mu \mathrm{m}$ particle sizes) was used for all analyses. A linear gradient of methanol $(50 \%)$ in water $(90 \%$ of acetonitrile) in the ratio of $10: 90$ and methanol (50\%) and acetonitrile (50\%) were used as a mobile phase with a flow rate $(1.5$ $\mathrm{ml} / \mathrm{min}$ ) for 10 min elution at room temperature. The injection volume of $25 \mu \mathrm{l}$ of the sample was injected to the HPLC for the test run. Solvents were pre-filtered before use by a Millipore filtration unit (USA). The analyses were carried out triplicate for each sample.

The absorbance of safranal at $320 \mathrm{~nm}$ and crocin at $440 \mathrm{~nm}$ was detected in UV-DAD. A calibration curve was prepared on the basis of the external standard using final concentrations of 10,20 and 30 $\mu \mathrm{g}$ concentrations of crocin and safranal in triplicate. Quantitative analyses were performed into account with respect to molecular coefficient absorbance for every peak acquired at the specific wavelength of maximum absorbance of the relevant compound (s) reported earlier. Concentrations of crocin and safranal are expressed in milligrams per gram (mg/g) of saffron stigmata.

\section{Calibration curve}

Stock solutions $(1 \mathrm{mg} / \mathrm{ml})$ of crocin and safranal were prepared in methanol, and 10-30 $\mu \mathrm{g}$ amounts of these compounds were used for the calibration curve to check the linearity.

High-performance liquid chromatography method validation and quantitative determination

The method was used to evaluate the content of crocin and safranal extracted from C. sativus stigma. Crocin and safranal samples were identified and quantified by comparison of retention time and absorbance on DAD-UV spectra with that of standard compounds. The quantitative analysis was studied on the basis of molecular coefficient absorbance of each peak found at the maximum absorbance (wavelength) of the crocin and safranal as previously reported. They are expressed in milligrams per gram of saffron stigmata on dry weight basis. The linearity of detector responses tested for both compound and repeatability of the method was confirmed by analyzing three samples of $C$. sativus.

\section{Statistical analysis}

All samples analyzed in triplicates for the accuracy of the result as a representational mean \pm standard error (SE).

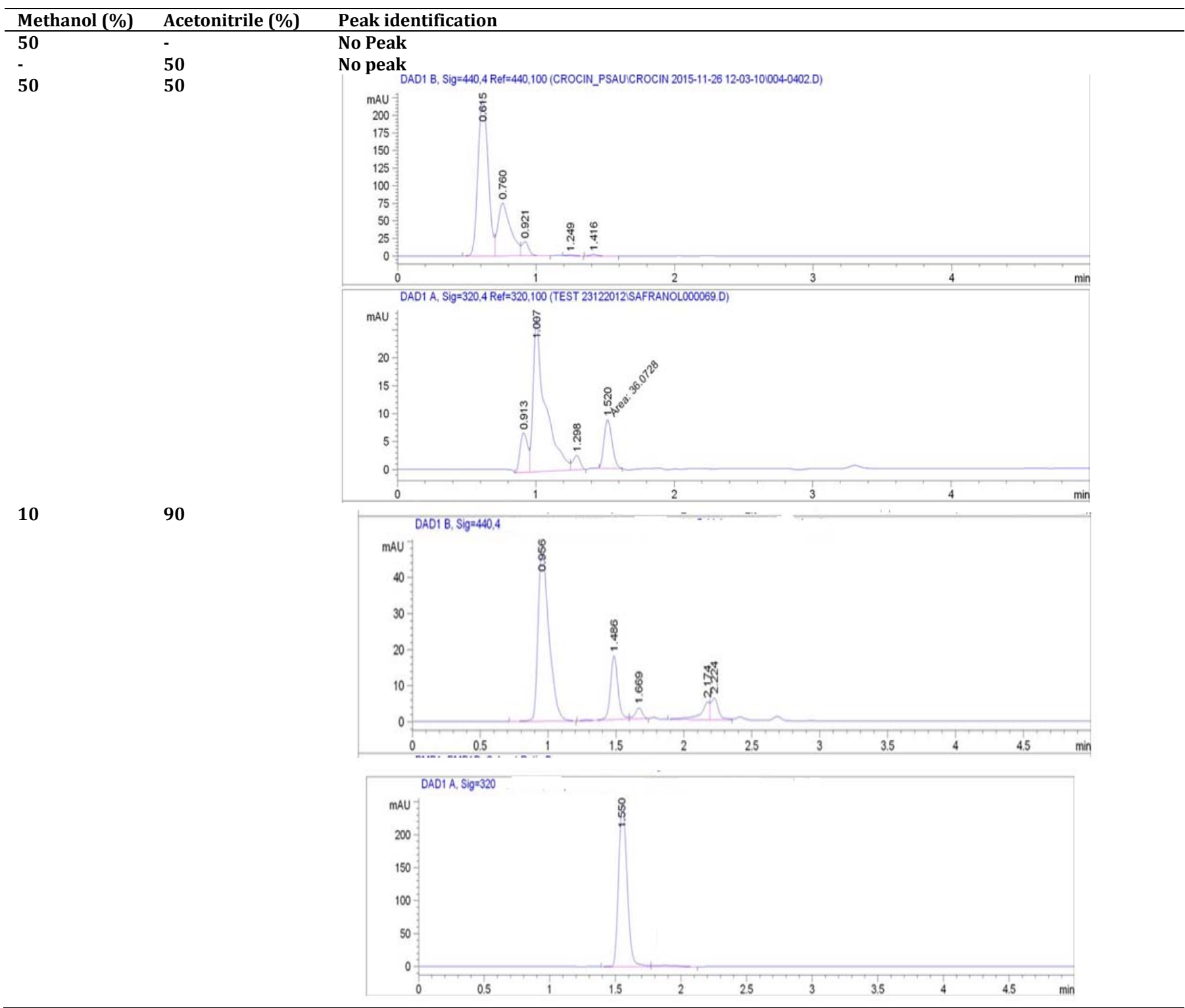

Fig. 1: Optimization of solvent (methanol and acetonitrile in aqueous phase) ratio for crocin and safranal for peak identification at particular wavelength $440 \mathrm{~nm}$ and $320 \mathrm{~nm}$ respectively 
Table 1: Parameters of calibration of crocin and safranal standards for HPLC (Each value is the mean \pm standard error $(n=3$ )

\begin{tabular}{|c|c|c|c|c|c|c|}
\hline Metabolite & Wavelength & Retention time & Linear regression & $\mathbf{R}^{2}$ & LOD & LOQ \\
\hline Crocin & 440 & 0.95 & $y=40.898 x+208.08$ & 0.9993 & $1.1 \pm 0.05$ & $3.4 \pm 0.10$ \\
\hline Safranal & 320 & 1.95 & $y=20.695 x+55.137$ & 0.998 & $4.2 \pm 0.06$ & $10.2 \pm 0.13$ \\
\hline
\end{tabular}

Table 2: Quantitative analyses of crocin and safranal present in market and SRC (Sara Alghonaim research chair) samples in stigma of Crocus sativus $L$. analyzed by HPLC (Each value is the mean \pm standard error $(n=3)$

\begin{tabular}{lll}
\hline Samples & Crocin content $\mathbf{~ m g} / \mathbf{g}$ & Safranal content mg/g \\
\hline T1 market & $15.41 \pm 0.02$ & $5.20 \pm 0.01$ \\
T2 market & $10.43 \pm 0.03$ & $5.19 \pm 0.03$ \\
T3 SRC & $16.32 \pm 0.02$ & $5.21 \pm 0.02$ \\
\hline
\end{tabular}

\section{RESULTS AND DISCUSSION}

The most commonly used separation technique in analytical science is HPLC estimated more than $65 \%$ recovery of the compound separated worldwide in the plant as well as another system [13]. The method of extraction by soaking plant material in the solvent was selected due to its simplicity and easy manageability. Methanol, water and ethanol were invoked as an extracting solvent because of their low toxicity and easy availability. The isolated crocin and safranal were first quantified one by one to record with their UV spectrum.

Two analyzed major compounds crocin and safranal were resolved under the chromatographic conditions. The conditions were thus obtained with an optimal chromatographic view after assay the different mobile phases (methanol, water and acetonitrile) with a reversed-phase C18 column in rapid HPLC system. Preliminary separation of the standard and the plant extract were estimated with a mobile phase consisting methanol and acetonitrile and $50 \%(\mathrm{v} / \mathrm{v})$ water using the gradient mode of elution. This mobile phase did not deliver sound resolution as in fig. 1. Thereafter, we modified the gradient mixture of a linear gradient of methanol $(50 \%)$ in water and acetonitrile (90\%) was used as a mobile phase with a flow-rate of $1.5 \mathrm{ml} / \mathrm{min}$ for a maximum elution time of $5 \mathrm{~min}$ at room temperature; the resolution was observed good.

The detection of crocin and safranal were performed by Diode Array Detector (DAD, noise levels of $< \pm 0.6 \mu A U / \mathrm{cm}$ the revolutionary $6 \mathrm{~cm}$ flow cell) gives up to 10 times higher sensitivity than other instruments using the 3D mode operation, allowing the collection of UV spectra of the constituents from 300 to $500 \mathrm{~nm}$ by steps of $4 \mathrm{~nm}$ in real time and checking the peak purity. Quantification of crocin and safranal were performed at a wavelength near their maximum UV absorbance wavelength. Crocin and safranal standards were quantified with fluorescence response at $440 \mathrm{~nm}$ and $320 \mathrm{~nm}$, respectively (fig. 1). To get the higher sensibility of detection of crocin and safranal, fluorescence mode was used to provide extracts retention time, DAD wavelength, range of quantification, and limit of detection (LOD) for all standards (table 1). There is no peak observed after $5 \mathrm{~min}$. But in previous reports retention time was observed after five minutes [4,14]. This is because of optimization of methods and new system quantification.

Calibration curve was constructed by using the corrected analyte concentrations with purity against the peak of the crocin and safranal. Crocin and safranal contents were quantified and calibration curves were produced. The $\mathrm{R}^{2}$ for the two standard curves is 0.999 with the slope R. SD values lower than $1.5 \%$. The range, of injected standards concentrations, were in between 10-30 $\mu \mathrm{g} / \mathrm{ml}$ for both safranal and $10-30 \mu \mathrm{g} / \mathrm{ml}$ for crocin. The calibration curves were passed through zero, and the contents of crocin and safranal were calculated using the regression equation obtained from the calibration curve.

A good linearity was achieved in the range of 10-30 $\mu$ g for each compound with the determination coefficient $\left(\mathrm{R}^{2}\right)$. The determination coefficient $\left(\mathrm{R}^{2}=0.999\right)$ for crocin and $\mathrm{R}^{2}=0.998$ for safranal were observed in our study (table 1). On the basis of standard crocin and safranal retention time, we have calibrated the yield percent recovery of the compounds in different plant extract (table. 2). The calibration curves exhibited linear regression (r.
0.997) for this rapid-HPLC method and LOQs were achieved in the range of $3.2 \mu \mathrm{g} / \mathrm{ml}$ for crocin and $10.2 \mu \mathrm{g} / \mathrm{ml}$ for safranal. The LODs for all standards were $\varangle .2 \mu \mathrm{g} / \mathrm{ml}$ which is consonant with other reports $[4,14-16]$.

\section{Analysis of a crude plant extract}

A methanol, ethanol and methanol: crude water extracts of $C$. sativus stigma was analyzed though HPLC and evaluated the separation efficiency of the crocin and safranal. Different combinations of methanol with water at 50-50\%, were evaluated in order to determine the extraction efficiency and to reach optimized solvent for extraction. In our study, we have used two solvent for extraction of crocin and safranal eg.; methanol: water gradient (50-50\%) and ethanol. We have observed that the good amount of crocin and safranal obtained in the extract of methanol and water gradient (50:50). However, on the basis of concentration used for methanol and water, the performed chromatographic method conducted a good separation and resolution of the sample components, better than other proposed gradient conditions reported previously [11,17-18].

To verify the rationality of our method, the crocin and safranal concentration was evaluated. The extraction yield proved to be very dependent on the nature of the compound. Crocin and safranal were completely retrieved from stigma. The range of crocin content (10.43-16.32 mg/g) and safranal (5.19-5.21 mg/g) was obtained in three samples (table 2). The proposed extraction method resulted in $99 \%$ recovery for all studied compounds (table 1 ).

\section{CONCLUSION}

The simple and specific HPLC method developed in this study can be used for the quality control of saffron in a quick way. This is interesting since it demonstrates that this method has the potential of good quantification of crocin and safranal efficiency and hence this can be used for other active compounds or precursors in C. sativus.

\section{ACKNOWLEDGMENT}

The authors acknowledge the financial support provided by Sara bint Rached bin Ghonaim Research Chair for Cultivating NonTraditional Medicinal and Aromatic Plants, Biology Department, College of Science and Humanities, Prince Sattam bin Abdulaziz University (PSAU), Alkharj, Kingdom of Saudi Arabia (KSA). The authors are thankful for Mr. A. Algorashy and Mr. A. Abou-Elkhair for their help in the field and lab work. The authors are also thankful for the Food and Agriculture COST Action FA1101 (Saffronomics) for the knowledge gained within this network.

\section{CONFLICT OF INTERESTS}

\section{Declared none}

\section{REFERENCES}

1. Tarantilis PA, Polissiou MG. Isolation and identification of the aroma components from saffron (Crocus sativus). J Agric Food Chem 1997;45:459-62.

2. Kanakis CD, Daferera DJ, Tarantilis PA, Polissiou MG. Qualitative determination of volatile compounds and quantitative evaluation of safranal and 4-hydroxy-2,6,6thimethyl-1-cyclohexene-1-carboxaldehyde (HTCC) in Greek saffron. J Agric Food Chem 2004;52:4515-21. 
3. Lozano P, Delgado D, Gomez D, Rubio M, Iborra JL. A nondestructive method to determine the safranal content of saffron (Crocus sativus L.) by supercritical carbon dioxide extraction combined with high-performance liquid chromatography and gas chromatography. J Biochem Biophys Meth 2000;43:367-78.

4. Zareena AV, Variyar PS, Gholap AS, Bongirwar DR. Chemical investigation of gamma-irradiated saffron (Crocus sativus L.). J Agric Food Chem 2001;49:687-91.

5. Alonso GL, Salinas MR, Esteban-Infantes FJ, Sanchez-Fernandez MA. Determination of safranal from saffron (Crocus sativus L.) by thermal desorption-gas chromatography. J Agric Food Chem 1996;44:185-8.

6. Sampathu SR, Shivashankar S, Lewis YS. Saffron (Crocus sativus L.): cultivation, processing, chemistry and standardization. Crit Rev Food Sci Nutr 1984;20:123-57.

7. Zalacain A, Ordoudi SA, Blazquez I, Diaz-Plaza EM, Carmona M, Blazquez MZI. et al. Near-infrared spectroscopy in saffron quality control: determination of chemical composition and geographical origin. J Agric Food Chem 2005;53:9337-41.

8. Narasimhan H, Chand H, Rajalakshmi D. Saffron: quality evaluation by sensory profile and gas chromatography. J Food Qual 1992;15:303-14.

9. Lozano P, Castellar MR, Simancas MJ, Iborra JL. Quantitative high-performance liquid chromatographic method to analyse commercial saffron (Crocus sativus L.) products. J Chromatogr A 1999;830:477-83.

10. Zougagh M, Rios A, Valcarcel M. An automated screening method for the fast, simple discrimination between natural and artificial colorants in commercial saffron products. Anal Chim Acta 2005;535:133-8.
11. Tarantilis PA, Tsoupras G, Polissiou M. Determination of saffron (Crocus sativus L.) components in crude plant extract using high-performance liquid chromatography-UV-Vis photodiode array detection mass spectrometry. J Chromatogr 1995;699:107-18

12. Alonso GL, Salinas MR, Garijo J, Sanchez-Fernandez MA. The composition of crocins and picrocrocin from Spanish saffron (Crocus sativus L.). J Food Qual 2001:24:219-33.

13. Boligon AA, Athayde ML. The importance of HPLC in the analysis of plants extracts. Austin Chromatography 2014;1:2.

14. Manzo A, Panseri S, Bertoni D, Giorgi A. Economic and qualitative traits of italian alps saffron. J Mountain Sci 2015;12:1542-50.

15. Zarinkamar F, Tajik S, Soleimanpour S. Effect of altitude on anatomy and concentration of crocin, picrocin and safranal in "Crocus sativus L." Aust J Crop Sci 2011;5:831-8.

16. Lage M, Cantrell CL. Quantification of saffron (Crocus sativus L.) metabolites crocins, picrocrocin and safranal for quality determination of the spice grown under different environmental moroccan conditions. Sci Hortic 2009;121:366-73.

17. Hadizadeh F, Mahdavi M, Emami SA. Evaluation of ISO method in saffron qualification. Acta Hortic 2009;739:405-10.

18. Castellar MR, Montijano H, Manjón A, Iborra JL. Preparative high-performance liquid chromatographic purification of saffron secondary metabolites. J Chrom 1993;648:187-90.

\section{How to cite this article}

- $\quad$ Pravej Alam, Shereen F Elkholy, Munetaka Hosokawa, Sabry A Mahfouz, Mahmoud A Sharaf-Eldin. Simultaneous extraction and rapid HPLC-based quantification of crocin and safranal in saffron (Crocus sativus L.). Int J Pharm Pharm Sci 2016; 8(10):224-227. 